\title{
LGR5 promotes the proliferation of colorectal cancer cells via the Wnt/ $\beta$-catenin signaling pathway
}

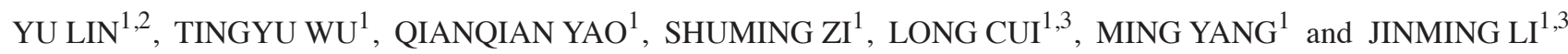 \\ ${ }^{1}$ Department of Colorectal Surgery, Xinhua Hospital Affiliated to Shanghai Jiaotong University School of Medicine, \\ Shanghai 200092; ${ }^{2}$ Department of Colorectal Surgery, Union Hospital, Fujian Medical University, Fuzhou, Fujian 350001; \\ ${ }^{3}$ Shanghai Colorectal Cancer Research Centre, Shanghai Jiaotong University School of Medicine, Shanghai 200025, P.R. China
}

Received May 11, 2014; Accepted February 2, 2015

DOI: $10.3892 / 01.2015 .3144$

\begin{abstract}
Leucine-rich repeat-containing G-protein coupled receptor 5 (LGR5) is an established cancer stem cell marker and is a target gene of the Wnt/ $\beta$-catenin signaling pathway, a critical pathway in the process of tumor initiation and growth. In the present study, the mRNA expression levels of LGR5, adenomatous polyposis coli (APC) and $\beta$-catenin were detected in 20 colorectal cancer (CRC) tissues and matched healthy mucosa samples using reverse transcription-quantitative polymerase chain reaction. HT-29 CRC cell line was treated with siRNA-Lgr5; the APC, $\beta$-catenin and LGR5 RNA expressions were detected and cell viability was measured using a CCK8 assay. The results revealed that LGR5 was significantly overexpressed in CRC tissue compared with healthy mucosa $(\mathrm{P}<0.05)$. Furthermore, knockdown of LGR5 by small interfering RNA decreased the expression of APC and $\beta$-catenin in HT29 colon cancer cells as well as inhibited the proliferation of HT29 cells. These findings demonstrated that LGR5 expression is critical for the promotion of neoplastic CRC cell proliferation, indicating that LGR5 may be a novel therapeutic target for CRC.
\end{abstract}

\section{Introduction}

Colorectal cancer (CRC) is one of the most common causes of cancer-associated mortality, accounting for $>600,000$ mortalities per year, worldwide (1). Tumor recurrence and metastasis to distant organs are the predominant contributing factors to the high mortality and poor survival

Correspondence to: Professor Ming Yang or Dr Jinming Li, Department of Colorectal Surgery, Xinhua Hospital Affiliated to Shanghai Jiaotong University School of Medicine, 1665 Kongjiang Road, Shanghai 200092, P.R. China

E-mail: yangming_6688@126.com

E-mail: jinmingli_dr@126.com

Key words: colorectal cancer, leucine-rich repeat-containing G-protein coupled receptor 5, Wnt/ $\beta$-catenin signaling, small interfering RNA rates associated with this disease (2). Recently, a number of studies have indicated that only a subpopulation of tumor cells, termed cancer stem cells (CSC), are capable of regenerating the tumor (3-5). In addition, CSCs may be involved in therapeutic resistance, tumor relapse and metastasis (3). Thus, the emergence of the CSC theory may have significant implications in cancer therapy. CSCs are considered to originate from mutant wild-type stem cells (6). In 2007, Barker et al (7) identified that leucine-rich repeat-containing $\mathrm{G}$ protein-coupled receptor 5 (LGR5) expression was restricted to cells in the crypt base of the small and large intestines; in addition, LGR5 was considered to be a stem cell marker. A subsequent study proposed that intestinal epithelial tumors may originate from LGR5-positive stem cells (8).

LGR5, also known as HG38 and $\mathrm{G}$ protein-coupled receptor $49(9,10)$, is a target gene of the $\mathrm{Wnt} / \beta$-catenin signaling pathway (11), acting as receptor for the $\mathrm{Wnt} / \beta$-catenin signaling agonist R-spondin $(12,13)$. This signaling pathway has a critical role in normal development and the maintenance of adult stem cells as well as in tumor pathogenesis and growth $(14,15)$. In healthy mucosa tissue, $\beta$-catenin is maintained at low cytoplasmic levels due to degradation regulated by a destruction complex composed of glycogen synthase kinase 3, Axin, adenomatous polyposis coli (APC) and other factors (16). In the progression of the majority of cases of CRC, the Wnt signaling pathway is activated early via truncations of APC and, less frequently, mutations of $\beta$-catenin (17). These mutations inhibit the activity of the destruction complex, resulting in the accumulation and nuclear translocation of $\beta$-catenin, ultimately resulting in transcriptional activation of target genes $(18,19)$. Nuclear $\beta$-catenin is involved in two processes that are essential for embryonic development: Epithelial-mesenchymal transition and stem cell formation (20). Accumulating data indicates that aberrant nuclear expression of $\beta$-catenin may confer these two traits to tumor cells, therefore driving malignant tumor progression $(21,22)$.

It is generally accepted that upregulation of LGR5 is associated with activated Wnt $/ \beta$-catenin signaling, resulting in the overexpression of LGR5 in various types of cancer, including hepatocellular carcinoma, ovarian cancer and CRC $(23,24)$. However, the underlying mechanisms for the role of LGR5 in carcinogenesis and intracellular signaling 
are poorly understood. Previous studies have identified that LGR5 and its homologs function as receptors of the R-spondin family of stem cell factors in order to enhance Wnt/ $\beta$-catenin signaling (25). Furthermore, knockdown of LGR5 induced cell death in adenoma and carcinoma cells (26); in addition, LGR5-positive stem cell fractions were capable of forming tumors via activation of the $\mathrm{Wnt} / \beta$-catenin signaling pathway (8). However, alternative studies propose that loss of LGR5 does not affect the proliferation or migration of intestinal cells (27). The aims of the present study were to further clarify the association between Lgr5, $\beta$-catenin and APC in the $\mathrm{Wnt} / \beta$-catenin signaling pathway and to identify a novel method for the treatment of colorectal cancer.

\section{Patients and methods}

Patients and specimens. Specimens were collected from 20 patients with $\mathrm{CRC}$ who underwent surgical resection at the Department of Colorectal Surgery of Xin Hua Hospital Affiliated to Shanghai Jiaotong University School of Medicine (Shanghai, China) between November 2010 and May 2013. Tumor and paired healthy adjacent colorectal mucosa tissue samples were collected from each patient. All samples were obtained from the surgically resected material, immediately frozen in liquid nitrogen (Novobio Scientific, Shanghai, China) and stored at $-80^{\circ} \mathrm{C}$. The current study was approved by the Ethics Committee of Shanghai Jiaotong University School of Medicine and all samples were obtained following receipt of written informed consent from all patients.

Reverse transcription-quantitative polymerase chain reaction $(R T-q P C R)$. RNA extraction was performed using TRIzol reagent (Invitrogen Life Technologies, Carlsbad, CA, USA), according to the manufacturer's instructions. Complementary DNA was then synthesized using SuperScript III Reverse Transcriptase (Invitrogen Life Technologies). Subsequently, qPCR was performed on a CFX96TM Real-Time System (Bio-Rad Laboratories, Inc., Hercules, CA, USA) using the following primers (Invitrogen Life Technologies): APC, F 5'-GCTCCAAGCCCAACCTTAA-3' and R 5'-GTTTTCGCCATCCACCAG-3'; $\beta$-catenin, F 5'-CAT TCAGCAGAAGGTCCGA-3' and R 5'-CTGGAAAACGCCATCACC-3'; and LGR5, F 5'-GTGGCAGCA AGTATGGCG-3' and R 5'-AGCAAAGGGAATTGAGCAAG-3'. Fold induction values were calculated using the cycle threshold $(\mathrm{Ct})$ method ( $\left.2^{-\Delta \Delta \mathrm{Ct}}\right)$ (28). All experiments were performed in triplicate and independently repeated a minimum of three times.

Small interfering RNA (siRNA). To knockdown LGR5, short hairpin RNA (shRNA) of the human LGR5 lentivirus gene transfer vector was constructed (Novobio Scientific). This gene transfer vector encoded the RNA sequence for green fluorescent protein (GFP). The following LGR5 siRNA sequence was used to target nucleotides: 5'-GTCTGCAATCAGTTACCTA-3'. Titer was measured by detecting GFP-positive HEK293T cells (Novobio Scientific) using fluorescence microscopy (IX51 microscope, Olympus Corporation, Tokyo, Japan), with the recombinant
Table I. T/N ratio of LGR5, $\beta$-catenin and APC mRNA expression.

\begin{tabular}{rrrr}
\hline Case & LGR5 & $\beta$-catenin & APC \\
\hline 1 & 2.73 & 6.37 & 0.58 \\
2 & 1.25 & 0.89 & 0.25 \\
3 & 0.76 & 1.65 & 3.32 \\
4 & 1.69 & 1.10 & 0.15 \\
5 & 3.10 & 1.16 & 0.46 \\
6 & 2.61 & 2.58 & 0.38 \\
7 & 2.03 & 0.98 & 1.07 \\
8 & 1.09 & 1.26 & 0.20 \\
9 & 20.47 & 0.99 & 0.32 \\
10 & 0.77 & 1.99 & 0.47 \\
11 & 5.53 & 1.86 & 0.31 \\
12 & 0.01 & 1.07 & 7.06 \\
13 & 0.03 & 2.86 & 0.28 \\
14 & 0.19 & 1.61 & 0.55 \\
15 & 9.64 & 0.86 & 0.49 \\
16 & 11.23 & 2.73 & 0.61 \\
17 & 4.03 & 0.66 & 0.48 \\
18 & 0.29 & 1.96 & 0.34 \\
19 & 1.38 & 1.46 & 0.06 \\
20 & 2.56 & 1.50 & 0.11 \\
& & & \\
\hline & & &
\end{tabular}

Values are presented as the $\mathrm{T} / \mathrm{N}$ ratio. $\mathrm{T} / \mathrm{N}$, tumor/normal healthy tissue expression; LGR5, leucine-rich repeat-containing G-protein coupled receptor 5; APC, adenomatous polyposis coli.

LGR5-targeting siRNA lentivirus prepared and titered to a concentration of $2.5 \times 10^{9}$ transfection units $/ \mathrm{ml}$. A scramble siRNA (Novobio Scientific) was used as a negative control (NC).

Detection of cell proliferation. NC-siRNA- and LGR5-siRNA-transfected cells were seeded into 96-well plates at a density of $4 \times 10^{3}$ cells/well and incubated for $24 \mathrm{~h}$. On days 1, 3, 5 and 7, $10 \mu \mathrm{l}$ Cell Counting Kit 8 (CCK8) solution (Dojindo Molecular Technologies, Inc., Shanghai, China) was added to each well. Color intensity was measured using an RT-2100C microplate reader (Rayto Life and Analytical Sciences Co.,Ltd., Nanshen, China) an absorbance of $450 \mathrm{~nm}$ to obtain cell growth curves. All experiments were performed in triplicate and repeated independently three times.

Statistical analysis. SAS software (version 8.5; SAS Institute, Inc., Cary, NC, USA) was used for all statistical analyses. Kruskal-Wallis non-parametric tests were performed to analyze differences in LGR5, APC and $\beta$-catenin mRNA expression between CRC and corresponding healthy mucosal tissues. In addition, a paired t-test was used to compare differences between the LGR5 knockdown group and negative control group. $\mathrm{P}<0.05$ was considered to indicate a statistically significant difference between values. 
A

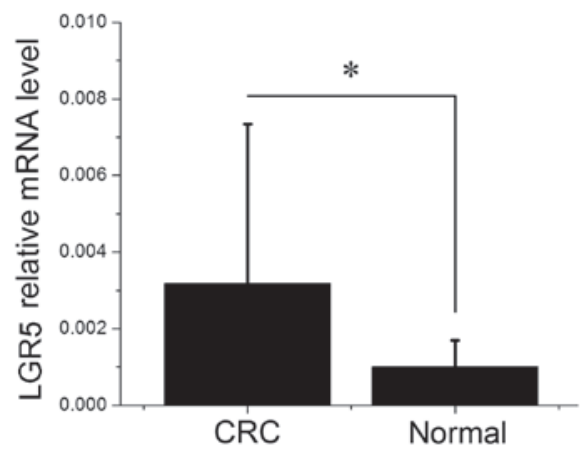

B

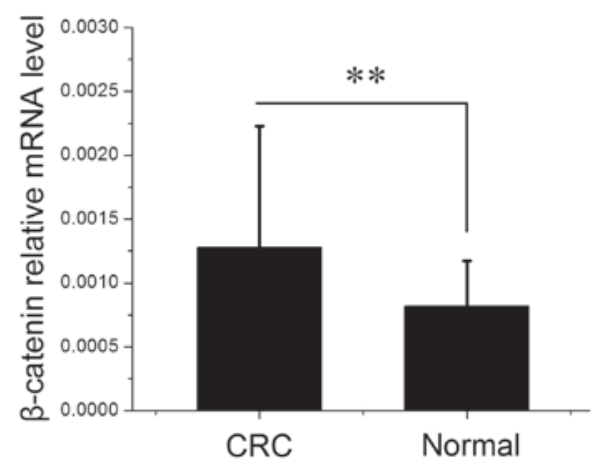

C

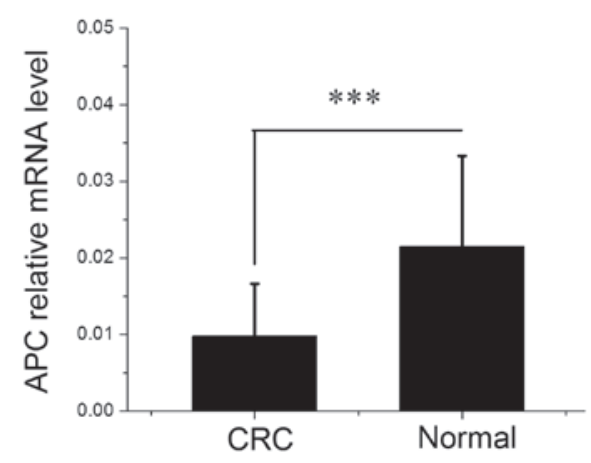

Figure 1. Relative mRNA expression levels of LGR5, $\beta$-catenin and APC in CRC tumor tissue and matched normal mucosa. (A) LGR5 and (B) $\beta$-catenin mRNA expression were significantly elevated in CRC compared with the matched normal mucosa. (C) APC mRNA expression was significantly decreased in the CRC samples. mRNA expressions levels were estimated by performing RT-qPCR and normalized to the expression of human $\beta$-actin Data are presented as the mean \pm standard deviation and significance was evaluated by performing Kruskal-Wallis non-parametric tests. All experiments were repeated independently three times. ${ }^{*} \mathrm{P}<0.05 ;{ }^{* *} \mathrm{P}<0.01$; ${ }_{* * * *} \mathrm{P}<0.001$. LGR5, leucine-rich repeat-containing G-protein coupled receptor 5; CRC, colorectal cancer; APC. adenomatous polyposis coli.

\section{Results}

$L G R 5$ and $\beta$-catenin expression is elevated and APC expression is reduced in CRC tissues. RNA was extracted from $20 \mathrm{CRC}$ and adjacent healthy tissues samples, then subjected to RT-qPCR to determine the mRNA expression profiles of LGR5, $\beta$-catenin and APC. As demonstrated in Fig. 1, there were significant differences in LGR5, $\beta$-catenin and APC mRNA expression levels between the CRC and healthy colorectal mucosa samples $(\mathrm{P}=0.0484,0.0032$ and 0.0006 , respectively). As shown in Table I, LGR5, $\beta$-catenin and APC expression in the CRC samples were divided by their expression in the matched healthy mucosa samples to obtain the tumor/normal healthy tissue expression $(\mathrm{T} / \mathrm{N})$ ratio.
A

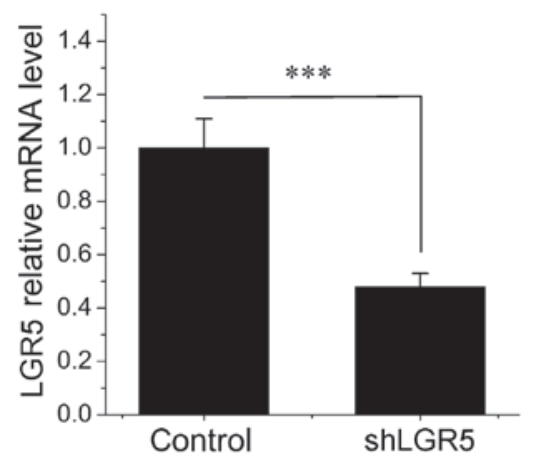

B

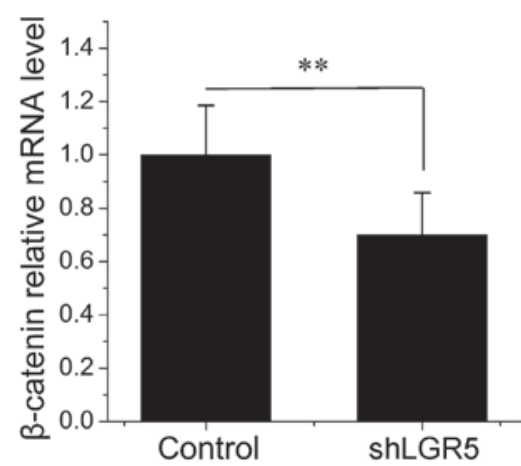

C

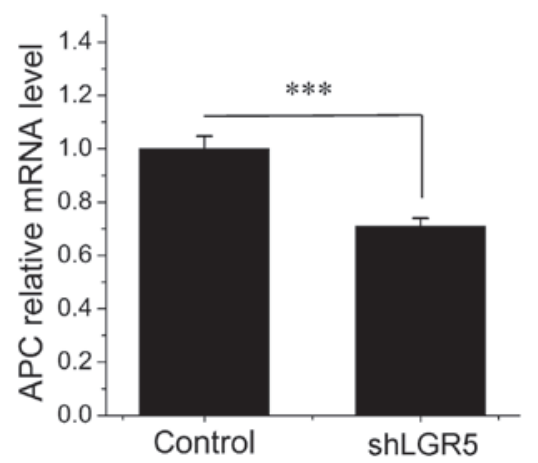

Figure 2. Relative mRNA expression levels of LGR5, $\beta$-catenin and APC in shLGR5-treated and control HT29 cells. (A) Knockdown experiments decreased LGR 5 mRNA levels by $52 \%$ compared with controls. Knockdown of LGR5 decreased (B) $\beta$-catenin and (C) APC mRNA expression by 30 and $29 \%$, respectively, compared with the controls. mRNA expression levels were estimated by RT-qPCR and expressed as fold changes relative to the control. Data are presented as the mean \pm standard deviation and significance was evaluated by performing t-tests, ${ }^{* * *} \mathrm{P}<0.01 ;{ }^{* * * *} \mathrm{P}<0.001$. All experiments were repeated independently three times. (sh)LGR5, (short hairpin RNA) leucinerich repeat-containing G-protein coupled receptor 5; APC. adenomatous polyposis coli.

A $\mathrm{T} / \mathrm{N}$ ratio of $>1$ indicated increased expression in CRC. Of the 20 CRC samples investigated, 14 (70\%) exhibited elevated levels of LGR5 expression and 15 (75\%) demonstrated elevated $\beta$-catenin expression compared with their corresponding healthy mucosa samples, with mean $\mathrm{T} / \mathrm{N}$ ratios of 3.57 and 1.78, respectively. By contrast, APC mRNA expression was decreased in $17(85 \%)$ CRC samples with a mean $\mathrm{T} / \mathrm{N}$ ratio of 0.87 .

siRNA-mediated knockdown of LGR5 inhibits the expression of APC and $\beta$-catenin. To investigate the functional relevance of LGR5 expression in CRC cell lines, the expression of LGR5 was knocked down in the HT-29 CRC cell line. Specific siRNA was constructed for LGR5 and its ability to knock down 


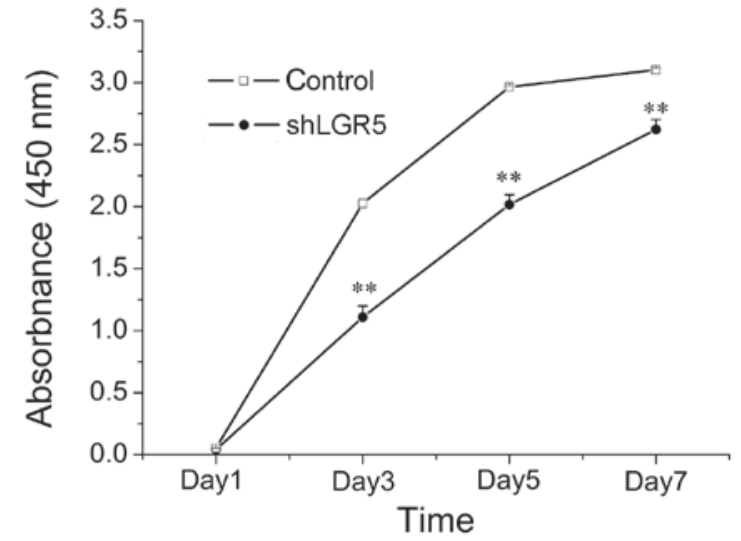

Figure 3. RNAi-mediated knockdown of LGR5 inhibits growth of HT29 cells in vitro. Knockdown experiments decreased Lgr5 mRNA levels by $52 \%$. Cell viability was measured using a CCK8 assay. Cells were counted every $48 \mathrm{~h}$. Data are shown as the mean \pm standard deviation. All experiments were repeated independently three times.** $\mathrm{P}<0.01$. (sh)LGR5, (short hairpin RNA) leucine-rich repeat-containing G-protein coupled receptor 5.

LGR5 mRNA was evaluated. RT-qPCR identified that the treatment of HT-29 cells with LGR5-siRNA resulted in a significant $52 \%$ decrease in LGR5 mRNA expression $(\mathrm{P}=0.0003)$ compared with the empty vector NC cells (Fig. 2A), indicating that the depletion of LGR5 using the siRNA method was effective. As illustrated in Fig. 2B and C, knockdown of LGR5 significantly decreased the expression of APC and $\beta$-catenin mRNA by $29 \%(\mathrm{P}=0.0003)$ and $30 \%(\mathrm{P}=0.001)$, respectively, compared with cells transfected with NC-siRNA at $48 \mathrm{~h}$ post-transfection. As APC is known to antagonize the transcriptional activity of $\beta$-catenin by promoting its nuclear export and its proteasomal destruction in the cytoplasm, decreasing the expression of APC may enhance Wnt/ $\beta$-catenin signaling (17-19). These results indicated that knockdown of LGR5 may inhibit the expression of $\beta$-catenin as well as promote $\beta$-catenin accumulation and nuclear translocation by downregulating APC.

Knockdown of LGR5 inhibits CRC cell proliferation. To investigate the effect of LGR5 on CRC cells viability, a viability curve of LGR5-knockdown HT29 cells was constructed by performing a CCK8 assay. As indicated in Fig. 3, HT29 cell growth was significantly inhibited following LGR5 knockdown compared with the growth of control group cells $(\mathrm{P}<0.001)$. This therefore indicated that downregulation of LGR5 expression using siRNA significantly inhibited the growth of HT-29 cells.

\section{Discussion}

LGR5, which has been established as a stem cell marker in the small intestine and colon, has also been identified as a downstream target gene of the Wnt signaling pathway (7). The current study demonstrated that LGR5 was significantly upregulated in CRC compared with healthy mucosa, which is comparable with the results of a number of previous studies $(24,29,30)$. The Wnt signaling pathway is comprised of a vast number of proteins, including APC and $\beta$-catenin, two proteins that are critical in CRC tumorigenesis (18). Therefore, the present study aimed to evaluate the association between LGR5, APC and $\beta$-catenin expression and CRC, as well as identify the role of LGR5 in Wnt signaling.

The mRNA expression levels of LGR5, APC and $\beta$-catenin were detected in $20 \mathrm{CRC}$ tissue samples and their corresponding healthy mucosa samples. The results demonstrated significant differences in LGR5, $\beta$-catenin and APC mRNA expression between CRC and healthy colorectal mucosa, indicating that CRC may be associated with aberrant activation of the $\mathrm{Wnt} / \beta$-catenin signaling pathway. In CRC, mutations in $\beta$-catenin, Axin and certain signaling pathways may result in the accumulation of $\beta$-catenin and enhance Wnt signaling activation (31). However, APC mutations, which result in aberrant activation of the Wnt signaling pathway, occur most frequently in CRCs (17).

In the present study, to understand the effects of LGR5 on APC and $\beta$-catenin expression, which are two key components of Wnt signaling, LGR5 expression was silenced in the HT-29 CRC cell line using siRNA. A decrease in APC and $\beta$-catenin mRNA expression was observed following knockdown of LGR5. These results indicated that LGR5 may be involved in regulating $\mathrm{Wnt} / \beta$-catenin signaling via modulation of the expression of APC and $\beta$-catenin. The role of APC and $\beta$-catenin in CRC tumorigenesis has been well studied. It was reported that $>90 \%$ of cases of CRC exhibit cytoplasmic accumulation of $\beta$-catenin (32). When activated and accumulated in the cytoplasm, $\beta$-catenin is transferred to the nucleus, where it activates numerous nuclear transcription factors, such as transcription factor (TCF)/lymphoid enhancer-binding factor, which results in the activation of downstream target molecules. Abnormal expression of these molecules may result in abnormal proliferation and tumorigenesis (33). APC is an important tumor suppressor that downregulates the transcriptional activity of $\beta$-catenin by the following three mechanisms: i) Reducing the levels of cytoplasmic $\beta$-catenin by binding to Axin; ii) promoting the export of nuclear $\beta$-catenin; and iii) sequestering $\beta$-catenin, preventing it from binding to TCF (34). The simultaneous decrease in APC and $\beta$-catenin expression observed in the present study provided evidence that LGR5 may mediate bidirectional regulation in the Wnt/ $\beta$-catenin signaling pathway ( $\beta$-catenin- or APC-directed signaling). Accumulating data has demonstrated that the silencing of LGR5 influences the functional and molecular outcome of CRC cells; for example, previous reports have indicated that knocking down endogenous LGR5 in cultured CRC cell lines reduced their proliferation, migration, growth rates and colony formation capability $(24,35,36)$. However, Walker et al (37) reported that the ablation of LGR5 increased invasion, induced anchorage-independent growth and enhanced tumorigenicity in a xenograft model. Based on these controversial results, the current authors proposed that LGR5 may act as a positive regulator of tumor growth when the $\beta$-catenin signaling pathway is predominant and acts as a negative regulator when the APC signaling pathway is predominant.

In the latter experiments of the present study, LGR5 downregulation resulted in the attenuation of HT29 cell proliferation. This data indicated that LGR5 may have a role in the regulation of CRC cell growth and proliferation, which is consistent with the results of 
previous investigations into basal cell carcinoma (15), Ewing sarcoma (24) and glioma (38). Thus, LGR5 may have the potential to serve as a therapeutic target in patients with CRC. However, future studies treating LGR5 as a therapeutic target should consider the bidirectional regulation of LGR5. Additionally, the current authors proposed that the blocking effect of LGR5 on APC may improve treatment efficiency.

In conclusion, the current results demonstrated that the majority of cases of CRC were associated with abnormal expression of LGR5, $\beta$-catenin and APC. Furthermore, knockdown of LGR5 significantly decreased the expression of $\beta$-catenin and APC. Due to the critical role of APC and $\beta$-catenin in colorectal tumor initiation and growth via the Wnt signaling pathway, LGR5 may be a potential therapeutic target for patients with CRC. However, the role of LGR5 in $\mathrm{Wnt} / \beta$-catenin signaling requires further investigation.

\section{Acknowledgements}

The present study was supported by grants from the Program of Shanghai's Subject Chief Scientist from Shanghai Municipal Health Bureau (no. XBR2011032), the Biomedical Engineering Research Funds of Shanghai Jiaotong University (no. YG2011MS32), the Special Fund for Outstanding Young Teachers of Shanghai Municipal Education Commission (no. JDY10112), the Ministry of Health (no. W2011JZC27) and Xinhua Hospital Affiliated to Shanghai Jiaotong University School of Medicine (no. 11YJ005).

\section{References}

1. Cunningham D, Atkin W, Lenz HJ, Lynch HT, Minsky B Nordlinger B and Starling N: Colorectal cancer. Lancet 375: 1030-1047, 2010.

2. Weitz J, Koch M, Debus J, Höhler T, Galle PR and Büchler MW: Colorectal cancer. Lancet 365: 153-165, 2005.

3. Visvader JE and Lindeman GJ: Cancer stem cells in solid tumours: Accumulating evidence and unresolved questions. Nat Rev Cancer 8: 755-768, 2008.

4. Odoux C, Fohrer H, Hoppo T, et al: A stochastic model for cancer stem cell origin in metastatic colon cancer. Cancer Res 68: 6932-6941, 2008.

5. Wu XS, Xi HQ, Chen L: Lgr5 is a potential marker of colorectal carcinoma stem cells that correlates with patient survival. World J Surg Oncol 10: 244, 2012.

6. Lobo NA, Shimono Y, Qian D and Clarke MF: The biology of cancer stem cells. Annu Rev Cell Dev Biol 23: 675-699, 2007.

7. Barker N, van Es JH Kuipers J, et al: Identification of stem cells in small intestine and colon by marker gene Lgr5. Nature 449: 1003-1007, 2007.

8. Barker N, Ridgway RA, van Es JH, et al: Crypt stem cells as the cells-of-origin of intestinal cancer. Nature 457: 608-611, 2009.

9. McDonald T, Wang R, Bailey W, et al: Identification and cloning of an orphan $\mathrm{G}$ protein-coupled receptor of the glycoprotein hormone receptor subfamily. Biochem Biophys Res Commun 247: 266-270, 1998.

10. Hsu SY, Liang SG and Hsueh AJ: Characterization of two LGR genes homologous to gonadotropin and thyrotropin receptors with extracellular leucine-rich repeats and a $\mathrm{G}$ protein-coupled, seven-transmembrane region. Mol Endocrinol 12: 1830-1845, 1998.

11. Van der Flier LG, Sabates-Bellver J, Oving I, et al: The intestinal Wnt/TCF signature. Gastroenterology 132: 628-632, 2007.

12. Carmon KS, Gong X, Lin Q, et al: R-spondins function as ligands of the orphan receptors LGR4 and LGR5 to regulate Wnt/beta-catenin signaling. Proc Natl Acad Sci USA 108: 11452-11457, 2011

13. de Lau W, Barker N, Low TY, et al: Lgr5 homologues associate with Wnt receptors and mediate R-spondin signalling. Nature 476: 293-297, 2011.
14. Early DS, Fontana L and Davidson NO: Translational approaches to addressing complex genetic pathways in colorectal cancer. Transl Res 151: 10-16, 2008.

15. MacDonald BT, Tamai K and $\mathrm{He} \mathrm{X}$ : Wnt/beta-catenin signaling: Components, mechanisms and diseases. Dev Cell 17: 9-26, 2009.

16. Cadigan KM and Peifer M: Wnt signaling from development to disease: Insights from model systems. Cold Spring Harb Perspect Biol 1: a002881, 2009.

17. Schneikert J and Behrens J: The canonical Wnt signalling pathway and its APC partner in colon cancer development. Gut 56: 417-425, 2007.

18. Clevers $\mathrm{H}$ and Nusse $\mathrm{R}$ : Wnt/ $\beta$-catenin signaling and disease. Cell 149: 1192-1205, 2012

19. Metcalfe $\mathrm{C}$ and Bienz M: Inhibition of GSK3 by Wnt signalling - two contrasting models. J Cell Sci 124: 3537-3544, 2011.

20. Brabletz T, Hlubek F, Spaderna S, et al: Invasion and metastasis in colorectal cancer: epithelial-mesenchymal transition, mesenchymal: Epithelial transition, stem cells and $\beta$-catenin. Cells Tissues Organs 179: 56-65, 2005.

21. Morin PJ and Weeraratna AT: Wnt signaling in human cancer. Cancer Treat Res 115: 169-187, 2003.

22. Taketo MM: Shutting down Wnt signal-activated cancer. Nat Genet 36: 320-322, 2004.

23. Yamamoto Y, Sakamoto M, Fujii G, et al: Overexpression of orphan G-protein-coupled receptor, Gpr49, in human hepatocellular carcinomas with beta-catenin mutations. Hepatology 37: 528-533, 2003.

24. McClanahan T, Koseoglu S, Smith K, et al: Identification of overexpression of orphan G protein-coupled receptor GPR49 in human colon and ovarian primary tumors. Cancer Biol Ther 5: 419-426, 2006.

25. Carmon KS, Lin Q, Gong X, et al: LGR5 interacts and cointernalizes with Wnt receptors to modulate Wnt/ $\beta$-catenin signaling. Mol Cell Biol 32: 2054-2064, 2012.

26. Al-Kharusi MR, Smartt HJ, Greenhough A, et al: LGR 5 promotes survival in human colorectal adenoma cells and is upregulated by PGE2: Implications for targeting adenoma stem cells with NSAIDs. Carcinogenesis 34: 1150-1157, 2013.

27. Garcia MI, Ghiani M, Lefort A, et al: LGR5 deficiency deregulates Wnt signaling and leads to precocious Paneth cell differentiation in the fetal intestine. Dev Biol 331: 58-67, 2009.

28. Livak KJ and Schmittgen TD: Analysis of relative gene expression data using real-time quantitative PCR and the 2(-Delta Delta C(T)) Method. Methods 25: 402-408, 2001.

29. Uchida H, Yamazaki K, Fukuma M, et al: Overexpression of leucine-rich repeat-containing $\mathrm{G}$ protein-coupled receptor 5 in colorectal cancer. Cancer Sci 101: 1731-1737, 2010.

30. Takahashi H, Ishii H, Nishida N, et al: Significance of Lgr5(+ve) cancer stem cells in the colon and rectum. Ann Surg Oncol 18: 1166-1174, 2011.

31. Yang M, Zhong WW, Srivastava N: G protein-coupled lysophosphatidic acid receptors stimulate proliferation of colon cancer cells through the $\{$ beta\}-catenin pathway. Proc Natl Acad Sci USA 102: 6027-6032, 2005.

32. Hsu HC, Liu YS, Tseng KC, et al: Overexpression of Lgr5 correlates with resistance to 5-FU-based chemotherapy in colorectal cancer. Int J Colorectal Dis 28: 1535-1546, 2013.

33. Hirsch D, Barker N, McNeil N, et al: LGR5 positivity defines stem-like cells in colorectal cancer. Carcinogenesis 35: 849-858, 2014.

34. Walker F, Zhang HH, Odorizzi A and Burgess AW: LGR5 is a negative regulator of tumourigenicity, antagonizes Wnt signalling and regulates cell adhesion in colorectal cancer cell lines. PLoS One 6: e22733, 2011

35. Tanese K, Fukuma M, Yamada T, et al: G-protein-coupled receptor GPR49 is up-regulated in basal cell carcinoma and promotes cell proliferation and tumor formation. Am J Pathol 173: 835-843, 2008

36. Fan XS, Wu HY, Yu HP, et al: Expression of Lgr5 in human colorectal carcinogenesis and its potential correlation with beta-catenin. Int J Colorectal Dis 25: 583-590, 2010.

37. Scannell CA, Pedersen EA, Mosher JT, et al: LGR5 is expressed by Ewing sarcoma and potentiates Wnt/ $\beta$-catenin signaling. Front Oncol 3: 81, 2013.

38. Wang D, Zhou J, Fan C, et al: Knockdown of LGR5 suppresses the proliferation of glioma cells in vitro and in vivo. Oncol Rep 31: 41-49, 2014. 